Pathologe 2012 · 33:477-478

DOI 10.1007/s00292-012-1699-0

Online publiziert: 23. Oktober 2012

(c) Springer-Verlag Berlin Heidelberg 2012

\section{Schmidt}

Referenzzentrum für Gynäkopathologie, Institut für Pathologie, Mannheim

\title{
Wie geht es weiter mit der gynäkologischen Zytologie in Deutschland?
}

Nach Einführung der gynäkologischen Krebsvorsorge als Leistung der gesetzlichen Krankenkassen für Frauen ab dem 20. Lebensjahr hat die Inzidenz des Zervixkarzinoms in Deutschland in beeindruckender Weise um etwa $70 \%$ abgenommen. Dieser starke Rückgang war umso erstaunlicher, wenn man berücksichtigt, auf welcher Basis sich dieser Erfolg zumindest in den ersten Jahren nach der Einführung des Screeningprogramms eingestellt hat.

In den Anfangsjahren war die gynäkologische Krebsvorsorge durch einige Unzulänglichkeiten charakterisiert. Eine Qualitätskontrolle heutiger Prägung fand nicht statt. Die Ausbildung der Zytologen erfolgte in vielen Institutionen ohne effektive Kontrolle. Eine zytologische Befundung der Präparate durch ZytologieAssistenten erfolgte nicht selten fernab vom Labor zu Hause. Die Liste der Unzulänglichkeiten ließe sich noch um weitere Punkte ergänzen, bis hin zu dem Vorwurf, dass in der gynäkologischen Zytologie auf vielfältige Weise versucht wurde (und nach wie vor wird), mit unerlaubten Mitteln wirtschaftlichen Erfolg zu sichern bzw. zu vergrößern.

Durch die Einführung der bundesweiten Qualitätsrichtlinien am 1. Oktober 2007 wurde zumindest ein Teil der Unzulänglichkeiten behoben. Die Änderungen betrafen die verbindliche Anwendung von Spatel und Bürste als Abnahmeinstrumente, die Einführung eines EDV-Systems mit Recall-System und eine
Jahresstatistik und die 2-jährliche Präparatekontrolle durch eine Qualitätskommission. Es ist schon jetzt erkennbar, dass die Einführung dieser Maßnahmen zu einer wesentlichen Qualitätssteigerung in der gynäkologischen Zytologie geführt hat. Die Häufigkeitsverteilung der verschiedenen Pap-Gruppen aus den jeweiligen Kassenärztliche Vereinigungen, die sehr ähnliche Werte ergeben, lässt dies vermuten, ebenso wie die persönlichen Erfahrungen des Autors aus der Arbeit der ZytologieKommission in Baden-Württemberg. PD Dr. Volker Schneider ist in seiner jüngst erschienenen Übersichtsarbeit zur aktuellen Situation der gynäkologischen Zytologie in Deutschland auf die Details und die Erfolge dieser Entwicklung eingegangen [1]. Besonders sind hierbei folgende Punkte hervorzuheben.

- Die Zahl technisch-inadäquater Präparate ist außerordentlich gering.

- Die Anzahl abklärungsbedürftiger zytologischer Befunde (rezidivierende Pap III D, Pap IVa) ist weitaus geringer als von Krankenkassenseite und einigen anderen Meinungsbildnern angegeben.

- Die Korrelation zytologischer und histologischer Befunde liegt bei der Gruppe IVa bei hervorragenden 79\%.

- Der positive prädiktive Wert der Gruppe IVa für die Diagnose einer zervikalen intraepithelialen Neoplasie(CIN)2-Läsion und höher (CIN 2+; unter Einschluss des Adenocarcinoma in situ, AIS) liegt bei $94 \%$.
Hinzu kommt nach einer Erhebung der Kassenärztlichen Bundesvereinigung (KBV) aus dem Jahre 2006, dass die Teilnahmerate an der gynäkologischen Vorsorgeuntersuchung, bezogen auf einen Zeitraum von 3 Jahren, bei 79\% liegt. Berücksichtigt man, dass dies ohne bürokratisch aufwendiges Einladungssystem möglich ist, spricht dies nicht nur für die Akzeptanz durch die weibliche Bevölkerung, sondern generell für den Erfolg dieses Programms.

Trotz der skizzierten Erfolge erlebt die Fachwelt und gelegentlich auch die Öffentlichkeit seit einigen Jahren eine Diskussion, die sowohl fachliche wie merkantile als auch gesundheitspolitische Aspekte beinhaltet. Es geht dabei um den Einsatz des HPV-Tests im primären Screening. Unbestritten ist, dass die Entwicklung der Zervixkarzinomvorstufen und des Zervixkarzinoms selbst, inklusive des typischen endozervikalen Adenokarzinoms, an eine Assoziation mit einer HPVInfektion gebunden ist; der Nachweis einer solchen also durchaus sinnvoll ist. Es ist aber ebenso unbestritten, dass nur $10 \%$ der Frauen, bei denen der HPV-Test positiv ausfällt, tatsächlich eine abklärungsbedürftige Läsion haben. Die Identifikation der Frauen mit einer derartigen Läsion gelingt demnach nicht allein mit dem HPVTest, dafür müssen zusätzliche Methoden herangezogen werden. Diese sind bislang entweder eine nachfolgende zytologische Untersuchung oder eine Differenzialkolposkopie. Verschiedene Studien haben 
jedoch gezeigt, dass bis zu $12 \%$ der HPVpositiven Fälle zytologisch negativ sind, die zytologische Untersuchung also auch nicht in allen Fällen weiterhilft. Die kolposkopische Untersuchung mit der Entnahme einer Biopsie hat ebenfalls keine $100 \%$ ige Sensitivität, was nicht selten durch die Schwierigkeit bei der Beurteilung der kleinen Biopsate begründet ist.

Entsprechend sind methodische Ansätze notwendig, mit denen es gelingt, eine behandlungsbedürftige Läsion treffsicherer zu erkennen, als das bisher der Fall ist. Der für dieses Ziel entwickelte und am besten untersuchte Test ist der Nachweis von p16 in den Zellen zervikaler intraepithelialer Neoplasien (CIN) und invasiver Karzinome, inklusive der Adenokarzinome, die in Zusammenhang mit einer HPV-Infektion entstehen. Der Test wird als alleiniger p16-Nachweis in der Immunhistochemie angeboten und als kombinierter p16/Ki67-Test in der Immunzytochemie. Eine klinische Validierung beider Testverfahren wurde von der Firma mtm laboratories AG (jetzt Roche mtmlabs AG, Mannheim) in aufwendigen und damit kostenträchtigen Studien durchgeführt, wobei die eingeworbenen finanziellen Mittel durch mehrere Investoren zur Verfügung gestellt wurden. Die Vergabe der Mittel ist allerdings auch während der Testentwicklung entgegen landläufiger Meinung kein Selbstläufer, sondern an die Erfüllung bestimmter sog. Milestones geknüpft, deren Erreichung nur durch die engagierte Mitarbeit aller Beteiligten möglich ist. Das erfolgreiche Zusammenspiel zwischen Investoren und Wissenschaftlern sollte dabei nicht Anlass für Kritik sein, sondern eher die Frage aufwerfen, ob solche Studien ohne die Industrie überhaupt möglich wären. Letztlich lässt in der aktuellen Situation die Ablehnung einer solchen Kooperation Zweifel dahin gehend aufkommen, ob eine Weiterentwicklung der gynäkologischen Zytologie überhaupt gewünscht ist.

\section{Wie kann diese Weiterent- wicklung konkret aussehen?}

Im Moment erscheint der Einsatz des neuen Testverfahrens nur in der Auswahl auffälliger zytologischer Befunde (Pap III/
IIW) und in Fällen eines Pap III D sinnvoll. Bei den auffälligen zytologischen Befunden könnte zunächst ein HPV-Test zum Einsatz kommen, der bei einem positiven Nachweis der High-risk-Typen 16 und 18 von einem p16/Ki67-Test gefolgt wird. Im Fall der anderen, weniger aggressiven HPV-high-risk-Typen könnte entweder der HPV-Test nach 6 Monaten kontrolliert werden oder die immunzytochemische Untersuchung mit p16/Ki67 erfolgen. In allen Fällen, in denen der p16/ Ki67-Test positiv ist, wäre eine Kolposkopie mit Biopsie anzuschließen.

Bei Fällen eines rezidivierten Pap III D sollte sofort ein p16/Ki67-Test durchgeführt werden, da $85 \%$ der Pap-III-DFälle HPV-high-risk-positiv sind. Bei Frauen mit unauffälligen zytologischen Befunden, jedoch positiven HPV-Test für HPV 16 oder 18, sollte unmittelbar eine Abklärung mit dem p16/Ki67-Test durchgeführt werden.

Mit dem konventionellen Pap-Abstrich, dem HPV-Test und dem p16/Ki67Test stehen heute Methoden zur Verfügung, die in der jeweiligen klinischen Situation an unter-schiedlichen Stellen in einem wissenschaftlich evidenzbasierten Algorithmus zum Einsatz kommen sollten. Es geht darum, ein bereits heute erfolgreiches Screeningprogramm, basierend auf dem Pap-Abstrich, weiter zu entwickeln und nicht mit der Einführung eines primären HPV-Screenings das Kind mit dem Bade auszuschütten. Angesichts der neuesten politischen Entwicklung erscheint dieser Wunsch allerdings wenig realistisch, ein Referentenentwurf zu einer Neuregelung der Vorsorgeuntersuchungen liegt dem Bundeskabinett vor. Dabei wird v. a. auf die Einführung eines Einladungssystems besonders hingewiesen. Nach Angaben des Bundesgesundheitsministeriums betragen die geschätzten Gesamtkosten für das Mammakarzinom, Kolonkarzinom und Zervixkarzinom 2366 Mio. EUR.

Inwieweit die HPV-Impfung einen Einfluss auf das Screening haben wird, steht noch nicht fest. Die Zahl der CIN2- und -3-Läsionen wird sehr wahrscheinlich deutlich zurückgehen. Daher wird zu überlegen sein, ob das Screeningintervall zu verändern ist. Allerdings sollte unter gar keinen Umständen auf ein regelmäßiges Screening verzichtet werden, da nicht alle Präkanzerosen und Karzinome von HPV 16 und 18, gegen die sich die Impfung richtet, verursacht werden. Hinzu kommt, dass es Adenokarzinome der Zervix gibt, die nicht HPV-assoziiert sind. Die jetzt regelmäßig erhobenen Jahresstatistiken der Kassenärztlichen Vereinigungen werden mögliche Änderungen im Spektrum der Pap-Gruppen anzeigen.

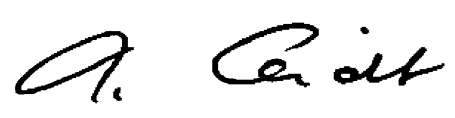

D. Schmidt

\section{Korrespondenzadresse}

Prof. Dr. D. Schmidt

Referenzzentrum für Gynäkopathologie, Institut für Pathologie

A 2, 2, 68159 Mannheim

schmidt@gyn-patho.de

Interessenkonflikt. Der korrespondierende Autor weist auf folgende Beziehung hin: Er ist Berater für die Fa. Roche Tissue Diagnostics.

\section{Literatur}

1. Schneider V (2012) Gynäkologische Krebsvorsorge in Deutschland. Gegenwärtiger Stand. Pathologe 33:286-292 